\title{
Long-term cumulative structural priming persists for (at least) one week
}

\author{
Michael P. Kaschak • Timothy J. Kutta • \\ Christopher Schatschneider
}

Published online: 10 November 2010

(C) Psychonomic Society, Inc. 2010

\begin{abstract}
We present an experiment that explores the degree to which cumulative structural priming effects of the sort reported in Kaschak (Memory and Cognition 35:925-937, 2007) persist over the course of a week. In the first session of the experiment, participants completed written sentence stems that were designed to bias them toward producing the double object (Meghan gave Michael a toy) or prepositional object (Meghan gave a toy to Michael) construction. Participants returned for a second session of the experiment a week later. We observed that the biases established in the first phase of the experiment affected performance in the second phase. That is, the cumulative priming effect persisted for a week. The implications of this result for theories of language production are discussed.
\end{abstract}

Keywords Language production - Structural priming ·

Syntax $\cdot$ Implicit learning $\cdot$ Linguistic adaptation

Structural priming refers to the tendency for language users to repeat syntactic structures across utterances (Bock, 1986). To illustrate using a particularly well-studied example, individuals who have recently heard or produced a double object construction (Meghan gave Michael a hug; DO) are more likely to subsequently produce another DO construction (The teacher sent the students a message) than to produce a prepositional object construction (The teacher sent a message to the students; PO). Structural priming is an extremely robust phenomenon. It has been observed in spoken (e.g., Bock, 1986) and written language production

M. P. Kaschak $(\varangle) \cdot$ T. J. Kutta • C. Schatschneider Department of Psychology, Florida State University, Tallahassee, FL 32306, USA

e-mail: kaschak@psy.fsu.edu (e.g., Cleland \& Pickering, 2006), with a range of syntactic constructions (e.g., Griffin \& Weinstein-Tull, 2003; Hartsuiker \& Kolk, 1998; Hartsuiker \& Westenberg, 2000;), in lab tasks (e.g., Bock, 1986; Pickering \& Branigan, 1998) and naturally occurring speech (e.g., Gries, 2005; Weiner \& Labov, 1983), and across several languages (e.g., Hartsuiker \& Kolk, 1998; Hartsuiker, Pickering, \& Veltkamp, 2004).

Structural priming is of interest to psycholinguists (and other cognitive scientists) for at least two reasons. First, the presence (or absence) of priming between individual prime and target sentences can be revealing with respect to the nature of the representations that underlie language production (see Pickering \& Ferreira, 2008, for an extensive review). For example, Bock, Dell, Chang and Onishi (2007) demonstrated that the structural priming effects that arise between language comprehension and language production follow the same pattern as the structural priming effects between sentences produced by the same individual. Bock et al.'s (2007) data suggest that language comprehension and language production may trade on the same or similar processing mechanisms. Second, it has been proposed that structural repetition effects can play a role in language acquisition (e.g., Brooks \& Tomasello, 1999; Savage, Lieven, Theakston, \& Tomasello, 2003) and language change (e.g., Chang, 2008). The tendency to repeat recently heard or produced constructions may help to both strengthen representations of a given structure (thus aiding the acquisition process; Tomasello, 2006), and (over time) alter the long-range probabilities of producing a given syntactic construction (such as the DO or PO), thereby contributing to language change (e.g., Bock \& Kroch, 1989; Chang, 2008). Studies of structural priming, therefore, have the potential to make broad contributions to our understanding of language. 
Two theories of structural priming have been advanced. Pickering and Branigan (1998) present an account based on a linguistic architecture in which nodes that represent lexical items (such as verbs) are linked to nodes that represent the rules governing the combination of words (e.g., the "noun phrase + prepositional phrase" rule licenses the production of the PO construction). When a sentence is produced, the link between the node representing the verb that is used (e.g., give) and the node representing the grammatical rule that is employed (e.g., "noun phrase + prepositional phrase") is activated. The activation that spreads in this network remains above baseline levels for several seconds (i.e., the node for the verb, the node for the combinatorial rule, and the link between those nodes maintains some residual activation), and the lingering activation produces structural priming. Because the mechanism of priming proposed by Pickering and Branigan (1998) is residual activation that decays within a few seconds, this theory leads to the prediction that structural priming effects should be relatively short-lived. Consistent with this prediction, short-lived priming effects have been reported in several studies (e.g., Branigan, Pickering, \& Cleland, 1999; Levelt \& Kelter, 1982; Wheeldon \& Smith, 2003).

Chang, Dell, and Bock (2006) offer an alternative view of structural priming in proposing that structural priming is the result of error-based learning within the language production system. Their account has been instantiated in a connectionist model that produces structural priming effects by allowing the model to continue to learn during the production of sentences. When a token of one construction (DO) is produced, the model weights are tuned such that it will be more likely to produce the same construction on a subsequent sentence. Chang et al. (2006) thus see structural priming as an example of implicit learning within the language production system (see also Bock \& Griffin, 2000; Ferreira, Bock, Wilson, \& Cohen, 2008). According to this account, it is expected that structural priming effects should be somewhat longlasting, as the change in model weights that result from choosing one construction over another (e.g., choosing the DO construction over the PO construction) will remain in place until the next time the same syntactic choice is faced. There are a number of reports of structural priming persisting in strength when several sentences intervene between the prime sentence and the subsequent target sentence (e.g., Bock \& Griffin, 2000; Hartsuiker, Bernolet, Schoonbaert, Speybroeck, \& Vanderelst, 2008).

Predictions about the duration of structural priming effects represent a major point of distinction between the extant theories of structural priming, and there have been a number of efforts to pin down the time course of structural priming over the last decade (e.g., Bock \& Griffin, 2000; Hartsuiker et al., 2008). The upshot of this work appears to be that the general structural priming effect can - but does not always - persist over several intervening sentences (e.g., Bock \& Griffin, 2000; Hartsuiker et al., 2008) and that particular aspects of the structural priming effect appear to be short-lived (e.g., the boost in priming that comes with the repetition of lexical items between prime and target sentences appears to decay rapidly; Hartsuiker et al., 2008). Although this work represents a good start toward understanding the duration of structural priming, continued development of our understanding of the temporal parameters of structural priming is of import for several reasons. First, whereas Chang et al.'s (2006) account can explain both short-lived and long-term priming effects, Pickering and Branigan's (1998) account cannot. Finding long-term priming effects (particularly priming effects over very long ranges of time) would thus be quite damaging to the Pickering and Branigan (1998) account. In addition, the finding of very long-range priming effects would fit with Bock and colleagues' contention that structural priming is an example of implicit learning within the language production system (Bock \& Griffin, 2000; Ferreira et al., 2008). This outcome would put constraints on the type of architecture that could be used as the basis for a general model of language production (e.g., by suggesting that language production is grounded in an error-driven learning mechanism such as the one employed by Chang et al., 2006). A second reason why it is important to understand the temporal parameters of structural priming is that, as mentioned above, structural priming has been proposed to play a role in language acquisition (Savage et al., 2003) and language change (Chang, 2008). However, understanding the extent to which structural priming can explain observations from language acquisition and language change depends on developing an understanding of the learning characteristics of the individual language user. For example, just how and over what range of time can the production of an individual sentence (or set of sentences) affect the production of subsequent utterances?

Although there is a large body of research that examines how the production of individual prime sentences affects the production of subsequent individual target sentences (see Pickering \& Ferreira, 2008, for a review), if studies of structural priming are going to contribute to a broader understanding of the nature of the language production system and its role in language learning and language change, it will be necessary to gain an understanding of how such effects operate over longer ranges of time and over larger bodies of language production experience. Some progress has been made in this regard. Bock and Griffin (2000) and Hartsuiker et al. (2008) both demonstrated that the priming between individual prime and target sentences is equally strong when the prime and target are produced on consecutive trials and when the prime and 
target are separated by as many as ten filler sentences. Kaschak and colleagues demonstrated both that the relative frequency with which particular constructions (such as the DO and PO) are produced earlier in an experiment affects the rate at which those constructions are produced later in the experiment (Kaschak, 2007; Kaschak \& Borreggine, 2008; Kaschak, Loney, \& Borreggine, 2006; see also Hartsuiker \& Westenberg, 2000) and that patterns of experience with particular verbs within particular constructions earlier in an experiment affect the rate at which those verbs are used in different constructions later in the experiment (Coyle \& Kaschak, 2008; see Bernolet \& Hartsuiker, 2010, for a related observation). Together, these data show that structural priming effects can persist and accumulate over relatively short periods of time - specifically, within the 30-60 minutes that it takes to run a typical experiment.

The study reported here is intended as a first step toward exploring how cumulative structural priming effects persist over a range of time that extends beyond the single-session experiments that have heretofore been reported in the literature. Our approach was to conduct an experiment that paralleled Experiment 1 reported by Kaschak (2007). The first phase of Kaschak's (2007) experiment (Bias phase) was designed to induce participants to produce a certain proportion of DO and PO constructions. The second phase of the experiment (Priming phase) allowed participants to produce DO or PO constructions at will in an effort to observe how patterns of experience in the Bias phase affected language production in the Priming phase. Kaschak's (2007) Experiment 1 used a written stem completion paradigm to demonstrate that the relative frequency of production of DO and PO constructions in the Bias phase affected the rates of production for those constructions in the Priming phase. Our goal was to assess whether this effect persists when the Bias phase and Priming phase of the experiment are separated by 1 week.

We entertained two broad possibilities for how this experiment might turn out. The first is a "return to normal" scenario wherein participants' language use (and exposure to the DO and PO constructions) during the 1 week between the Bias and Priming phases returns to its normal base rates in terms of the rate of production of the target constructions, thereby eliminating the effect of the Bias phase on the patterns of production seen in the Priming phase. The return-to-normal outcome would be reflective of learning in the language production system that is relatively insensitive to individual contexts of language use. The Bias phase establishes a particular skew in the rate of production for the DO and PO constructions. Once the participants leave the experiment, their ongoing language experience continues to shape their bias toward the DO or PO construction, overwriting the bias established within the experiment. ${ }^{1}$ That is, in tracking the probabilities of use of particular constructions, the language production system does not differentiate between experiences that occur within different contexts (e.g., the experiment and the various contexts the participants experience in their lives outside the experiment), and thus the effects of the Bias phase do not persist until the participants return to complete the Priming phase.

The second possibility is that the effect of the Bias phase will persist for more than 1 week and subsequently affect performance on the Priming phase. It has been argued that structural priming is an example of implicit or procedural learning (e.g., Chang et al., 2006; Ferreira et al., 2008), and implicit/procedural learning effects have been shown to persist for very long durations (e.g., Allen \& Reber, 1980; Kolers, 1976; Mitchell, 2006; Tulving, Schacter, \& Stark, 1982; Tunney, 2003). Although the participants' linguistic experience in the 1 week between the Bias and Priming phases of the study will likely expose them to a normal balance of DO and PO constructions, the literature on implicit learning suggests that the reinstatement of the experiment context will produce the conditions under which the participants' earlier experience in the experiment (Bias phase) will affect their behavior in the current phase of the study (Priming phase; see Allen \& Reber, 1980; Kolers, 1976; Mitchell, 2006; Tunney, 2003). That is, learning in the language production system is sensitive to patterns of behavior in different contexts of language use, and reinstating the context of the experiment will lead the participants' language production choices to reflect their experience in the first session of the experiment.

Our experiment paralleled Kaschak's (2007) Experiment 1. In the Bias phase of the study, participants were asked to complete written sentence stems (prime stems) designed to elicit either the DO (Meghan gave Michael...) or PO (Meghan gave a toy...) construction. In the Priming phase of the study, participants were asked to complete sentence stems (target stems) that allowed completion as either a DO or PO construction (Emma sent...). Beyond this, several modifications to the design of Kaschak's (2007) study were made. First, the experiment was split into two sessions, with the Bias phase occurring in the first session and the Priming phase occurring in the second session 1 week later. Second, because of the demands involved in bringing participants back for a second session of the experiment, we did not include all of the Bias phase conditions from Kaschak's (2007) original study. The present study

\footnotetext{
${ }^{1}$ Bock and Griffin (2000) estimate that speakers of American English would typically experience a $2 / 1$ split in favor of the DO construction. This is likely to be a reasonable estimate of the relative proportion of DO and PO constructions that our participants would experience in their life outside of our experiments.
} 
employed two Bias phase conditions - one in which participants' language production experience was biased $100 \%$ toward the DO construction (100\% DO condition) and one in which participants' experience was biased $100 \%$ toward the PO construction (100\% PO condition). As these conditions produced strong cumulative priming effects in Kaschak's (2007) experiment, we decided that the use of these two conditions would provide a sufficient test of whether or not cumulative priming effects from the Bias phase would persist for more than 1 week.

\section{Method}

Participants The participants were 40 introductory psychology students. They received partial course credit in exchange for their participation. As in previous experiments conducted using this paradigm, we wanted to be sure that participants' behavior in the Bias phase reflected the intended structural manipulations. Participants were excluded from the data analysis if their performance in the Bias phase of the experiment did not show a skew of at least 80 / $20 \%$ in favor of the dominant construction for their training condition (i.e., in the DO Bias condition, participants had to produce at least $80 \%$ DO constructions; otherwise, their experience in the Bias phase was considered to be too deviant from the intended proportion of DO and PO constructions). No participants were excluded based on this criterion.

Materials For the Bias phase, there were 20 pairs of prime stems, such that one member of each pair disposed participants to produce a DO completion (The swimmer handed the diver...) and the other member disposed participants to produce a PO completion (The swimmer handed the towel...). For the Priming phase, 20 critical target stems (The pianist sent...) that could be completed as either a DO or PO construction were created (see Appendix A). An additional 130 filler stems were constructed for use in the experiment. These stems were designed to elicit a range of transitive and intransitive constructions and were structured so that they could not easily be completed as a DO or PO construction. Eighty of the filler stems were assigned to appear in the Bias phase of the experiment, and the remaining filler stems were assigned to appear in the Priming phase.

Procedure Participants were randomly assigned to be in the $100 \%$ DO or $100 \%$ PO Bias phase condition (20 participants in each condition). They were told that they were going to see a series of sentence stems and that they should complete each one to make a grammatical English sentence. Once they had completed the sentence, they were to hit the
"RETURN" key on the keyboard in order to move on to the next stem. In the $100 \%$ DO condition, participants completed 20 DO-eliciting prime stems, while in the $100 \%$ PO condition, participants completed 20 POeliciting prime stems. Throughout the experiment (Bias and Priming phases), four or five filler stems separated each critical prime or target stem. Upon completing the Bias phase of the experiment, participants were told that the first phase of the study was completed and that they would need to be assigned a time to complete the second phase of the study. Participants were assigned a time slot around 7 days from the time of their participation in the Bias phase of the study. All participants returned for the Priming phase at least 6 days after the Bias phase. On average, participants completed the Priming phase of the study 7 days after the Bias phase. Upon returning for the Priming phase of the study, participants again engaged in the written stem completion task. Each participant completed six target stems that could be finished as either a DO or PO construction. That is, each participant only saw a random subset of six of the 20 target stems that were created for this phase of the experiment. We did this (rather than give all participants the same 6 target stems) in an effort to broaden the number of stimuli that were used in the Priming phase of the study while not unduly increasing the overall length of the experiment. The order of the prime and target stems seen throughout the experiment was randomized for each participant.

Scoring Stem completions were scored as follows. For prime stems, completions were scored as a DO if the completion was a noun phrase incorporating the patient of the verb. Completions were scored as a PO if they began with a prepositional phrase using the word "to" that incorporated the beneficiary of the verb. Target completions were scored as a DO if they consisted of two noun phrases, the first denoting the beneficiary of the verb and the second denoting the patient of the verb. Completions were scored as a PO if they consisted of a noun phrase denoting the patient of the verb and a prepositional phrase using the word "to" that denoted the beneficiary of the verb. All other completions, including completions that contained a verb particle (Meghan gave the toy back to Michael) and completions that were non-reversible (e.g., a PO completion that would not produce a grammatical DO completion: The girl gave it to her mom) were scored as "other."

Design and analysis The responses in the Bias phase were examined to ensure that performance fell within the target range of performance for that condition (as described earlier). The proportion of DO and PO constructions produced by each participant was calculated by dividing the number of responses of each type by the total number of 
DO and PO completions produced. This computation ignored trials on which "other" responses were produced. All participants' rates of DO and PO production fell within the range intended for their Bias phase condition (as described earlier).

The target completions from the Priming phase of the experiment were analyzed as follows. We first excluded any trials on which an "other" response was made (51 of 240 total trials in the experiment; $21 \%$ ). The proportion of "other" responses did not differ across Bias conditions $[t(238)=1.53, p=0.127]$. This resulted in a binary dependent variable (target completions being either a DO or PO, where DO was coded as 1 and PO coded as 0 ) that was analyzed using a cross-classified mixed logit model (see Jaeger, 2008), with participants and items serving as crossed random factors and Bias condition serving as a binary fixed factor (where DO Bias was coded as 1, and PO Bias was coded as 0 ). Intercepts were allowed to vary for both participants and items. Mixed logit models analyzing both the proportion of "other" responses in the preliminary analyses and the DO and PO stem completions on the remaining trials were run using the HLM statistical software (Raudenbush, Bryk, Cheong, \& Congdon, 2004). ${ }^{2}$ We report the model estimates for the fixed effects in the text and Table 1. Because they are of little theoretical interest, estimates of the random effects are presented in Appendix B.

\section{Results}

The proportion of DO and PO constructions produced in each Bias phase condition closely matched the intended proportions: in the $100 \%$ DO condition, participants produced 94\% DO completions, and in the 100\% PO condition, participants produced $98 \%$ PO completions.

Mixed logit model analysis of the target responses was performed to predict the logit-transformed likelihood of a DO target completion. The results of this analysis are presented in Table 1. The critical result of the analysis is a significant effect of the Bias condition $[t(187)=3.63$, $p=0.001]$. Participants were more likely to produce a DO

\footnotetext{
${ }^{2}$ The HLM software provides $t$-values assessing the statistical significance of model parameters, rather than the Wald $Z$ that is often reported with mixed logit models. Whereas the $t$-statistic and Wald $Z$ are asymptotically equivalent with large numbers of upper level units in a mixed models analysis (i.e., they yield essentially the same results), with smaller numbers of upper level units in the analysis, the $t$-statistic is more conservative. Thus, the HLM software employs the $t$-statistic, and we consequently report the $t$-statistic throughout this paper.
}

target completion in the DO Bias condition (0.66) than in the PO Bias condition (0.34). The odds ratio associated with the Bias condition effect was 4.54 , indicating that participants were fourfold more likely to produce a DO target completion in the DO Bias condition than in the PO Bias condition. The results of our experiment therefore show that the cumulative priming effect established in the Bias phase of the experiment affected language production in the Priming phase after a delay of 1 week.

\section{Discussion}

The goal of this work was to assess the extent to which the cumulative structural priming effects observed in previous studies would persist for long stretches of time, particularly a delay of 1 week. The result of our experiment is straightforward. Replicating the results of Kaschak's (2007) Experiment 1, we found that our participants were more likely to produce a DO target completion after having experienced the DO Bias condition 1 week prior, than after having experienced the PO Bias condition 1 week prior. In other words, the cumulative priming effect could be observed after a delay of 1 week.

The results of our experiment are in accord with the second possibility outlined in the Introduction of this article, namely, that the effects of the Bias phase would be long-lasting. As such, the data reported here are consistent with the notion that language producers can track the probability of use for particular constructions within different contexts of language use. The data are also generally consistent with the concept that structural priming is an example of implicit/procedural memory in the language production system (Chang et al., 2006; Bock \& Griffin, 2000; Ferreira et al., 2008). Just as implicit learning effects in other domains have been shown to persist over long stretches of time (e.g., Allen \& Reber, 1980; Kolers, 1976; Masson, 1984; Mitchell, 2006; Tulving et al., 1982; Tunney, 2003), the effect of learning structural biases in the Bias phase of these experiments could be observed over 1 week after the initial learning episode.

We have only explored one of the many possibilities for observing long-range priming effects within and between different kinds of language production tasks and, as such, it may be premature to propose a specific theoretical account of the data presented here. At the very least, it would be useful to know whether the longrange persistence of cumulative priming effects can be observed with different methods in the Bias and Priming phases (e.g., spoken production tasks), different structural alternations (e.g., active vs. passive), and a broader range of verbs than we employed in our experiment. It 
Table 1 Results of Experiment 1

\begin{tabular}{|c|c|c|c|c|c|c|}
\hline \multicolumn{7}{|c|}{ Mixed logit model results } \\
\hline Predictor & Coefficient & SE & $t$-value & $d f$ & $p$-value & Odds Ratio \\
\hline Intercept & -0.75 & 0.34 & -2.19 & 187 & 0.029 & 0.47 \\
\hline Bias condition & 1.51 & 0.42 & 3.63 & 187 & 0.001 & 4.54 \\
\hline \multicolumn{7}{|c|}{ Counts and Proportions of DO and PO Responses } \\
\hline & \multicolumn{2}{|l|}{ Raw Counts } & \multicolumn{2}{|l|}{$\%$ DO } & \multicolumn{2}{|c|}{ Estimated \% DO } \\
\hline Response Type: & DO & $\mathrm{PO}$ & & & & \\
\hline DO Bias Condition & 60 & 35 & .66 & & .68 & \\
\hline PO Bias Condition & 31 & 63 & .34 & & .32 & \\
\hline
\end{tabular}

Note: Estimated \% DO computed using model parameters

would also be important to know whether the cumulative priming effects observed using different paradigms persist as strongly as they did in our current paradigm. Although the data presented here do not provide us with the latitude to speculate broadly on the ways that these variables may affect long-range structural priming effects, we believe that an examination of these issues will be important for the further development of our understanding of the ways that principles of learning and memory contribute to language production.

The data reported here have implications for the two major theories that have been proposed to explain structural priming, namely, Pickering and Branigan's (1998) residual activation account and Chang et al.'s (2006) error-driven learning account. Pickering and Branigan's (1998) account has not yet been extended to long-range priming effects (even those within the course of a single experiment), and the demonstration that structural priming effects can persist for more than 1 week is likely to be a phenomenon that is difficult to explain for a model that accounts for priming via a mechanism of transient residual activation. It remains to be seen whether a version of Pickering and Branigan's (1998) approach can be adapted to handle long-range priming effects.

Our data are broadly consistent with Chang et al.'s (2006) assertion that structural priming is a form of implicit learning and, therefore, is potentially very long-lasting. Although we see our results as generally consistent with this approach, it should be noted that Chang et al.'s (2006) model has not yet been extended to look at structural priming over delays as long as ours, and so it remains to be seen whether their model would be able to successfully produce the results seen here. Furthermore, we are presuming that one reason why the structural priming effect did persist for more than 1 week is that participants were able to keep their rates of production for the DO and PO constructions in the experiment somewhat separate from their rates of production for the constructions in other contexts of language use. This sort of context-specific tracking of language behavior has not yet been captured in Chang et al.'s (2006) model (or, for that matter, in Pickering
\& Branigan's, 1998 approach). We believe this will be an interesting direction in which to extend theories of language production.

On a broader level, we see a connection between the "contexts of language use" issue raised here and the literature on conversational pacts (e.g., Brennan \& Clark, 1996; Clark, 1996; Pickering \& Garrod, 2004). That conversational partners develop local norms of communication during interactions has been well demonstrated (e.g., Brennan \& Clark, 1996; Garrod \& Doherty, 1994). In addition, whereas the long-term memory properties of these norms have not been explored over time spans of the sort considered in our experiments, it is not unreasonable to surmise that these adaptations would function in manner similar to what was observed here: the development of local norms may not necessarily change a person's linguistic behavior in the broad sense (i.e., once conversational partners leave a specific dialogue; Metzing \& Brennan, 2003), but it may strongly affect the person's behavior if he/ she returns to the same general context of language use (e.g., having a conversation with the same partner). We believe that an exploration of these issues will be essential to discerning how linguistic experience within particular contexts affects subsequent language behavior in similar and different contexts. Understanding these issues will be key to gaining a sense of how structural priming and other conversational adaptation effects contribute to phenomena in language development and language change.

The experiment presented here represents a first step toward examining the extent to which cumulative structural priming effects persist over longer ranges of time than have been considered in previous studies. Clearly, we have only scratched the surface in terms of studying long-range learning and adaptation effects in language production. It is our hope that this experiment will spur more interest in exploring the ways that mechanisms of learning and memory contribute to language production on different time scales, from the short-range priming that occurs between prime and target sentences to the long-range learning effects that shape broad patterns of language usage. 


\section{Appendix A}

Prime and target stems used in Experiment 1.

Prime Stems (DO eliciting version presented first)

1) The swimmer handed the diver/The swimmer handed the towel

2) The good looking bartender gave the girls at the bar/ The good looking bartender handed free drinks

3) The millionaire gave the struggling artist/The millionaire loaned the valuable painting

4) The unhappy customer sent the business owner/The unhappy customer handed the long fax

5) The woman sent the insurance company/The woman handed the claim

6) The builder handed his new client/The builder loaned the blueprints

7) The lecturer loaned the professor/The lecturer lent the book

8) The architect sent the president of the company/The architect gave the model of the building

9) The grandmother handed the little girl/The grandmother gave the big present

10) The photographer loaned the editor/The photographer sent the finished proofs

11) The researcher sent the experienced surgeon/The researcher sent the detailed results

12) The happy child gave her father/The happy child handed the coloring book

13) The captain handed the old sailor/The captain handed the spare life jacket

14) The eager boyfriend handed his girlfriend/The eager boyfriend gave the box of flowers

15) The secretary gave the manager/The secretary handed the invoice

16) The mean neighbor lent the woman next door/The mean neighbor gave a nasty note

17) The travel agent lent the young fan/The travel agent handed the last ticket

18) The ship's captain handed the admiral/The ship's captain lent her travel log

19) The mother handed the hungry toddler/The mother gave the expensive toy

20) The young couple sent the IRS investigator/The young couple sent their mortgage payment

\section{Target Stems}

1) The politician sent

2) The librarian lent

3) The famous novelist sent

4) The consultant loaned

5) The mailman gave

6) The kidnapper sent
7) The pianist sent

8) The fisherman gave

9) The investigator lent

10) The policeman handed

11) The worker lent

12) The businessman sent

13) The runner handed

14) The woman gave

15) The fireman handed

16) The diver handed

17) The writer gave

18) The owner lent

19) The artist handed

20) The repairman loaned

\section{Appendix B}

The estimates for random effects of participants and items in our regression analysis are presented below.

\begin{tabular}{llllll}
\hline & Std. Dev & $\begin{array}{l}\text { Variance } \\
\text { Component }\end{array}$ & $d f$ & $\mathrm{X}^{2}$ & $p$-value \\
Participants & 0.74 & 0.55 & 38 & 57.74 & 0.02 \\
Items & 0.82 & 0.68 & 18 & 43.14 & 0.001 \\
\hline
\end{tabular}

In both cases, the analyses indicate significant variability associated with differences in performance across participants and items. Differences across items have been noted in other experiments, including those reported by Kaschak (2007), and likely reflect (among other things) interactions between existing verb biases and the biases induced within our experiments. Variability in priming effects across participants has not been widely explored. In keeping with the hypothesis that structural priming is an example of implicit learning, one explanation for the variability across participants is that it is driven by variability in implicit learning abilities (see Kaufman, Deyoung, Gray, Jiminez, Brown, \& Mackintosh, 2010, for a discussion).

\section{References}

Allen, R., \& Reber, A. S. (1980). Very long-term memory for tacit knowledge. Cognition, 8, 175-185.

Bernolet, S., \& Hartsuiker, R. J. (2010). Does verb bias modulate syntactic priming? Cognition, 114, 455-461.

Bock, J. K. (1986). Syntactic persistence in language production. Cognitive Psychology, 18, 355-387.

Bock, J. K., \& Kroch, A. S. (1989). The isolability of syntactic processing. In G. N. Carlson \& M. K. Tanenhaus (Eds.), Linguistic structure in language processing. Dordrecht: Reidel.

Bock, J. K., \& Griffin, Z. M. (2000). The persistence of structural priming: Transient activation or implicit learning? Journal of Experimental Psychology: General, 129, 177-192. 
Bock, K., Dell, G. S., Chang, F., \& Onishi, K. (2007). Structural persistence from language comprehension to language production. Cognition, 104, 437-458.

Branigan, H. P., Pickering, M. J., \& Cleland, A. A. (1999). Syntactic priming in written production: Evidence for rapid decay. Psychonomic Bulletin \& Review, 6, 635-640.

Brennan, S. E., \& Clark, H. H. (1996). Conceptual pacts and lexical choice in conversation. Journal of Experimental Psychology. Learning, Memory, and Cognition, 22, 1482-1493.

Brooks, P. A., \& Tomasello, M. (1999). Young children learn to produce passives with nonce verbs. Developmental Psychology, 35, 29-44.

Chang, F. (2008). Implicit learning as a mechanism of language change. Theoretical Linguistics, 34, 115-122.

Chang, F., Dell, G. S., \& Bock, K. (2006). Becoming syntactic. Psychological Review, 113, 234-272.

Clark, H. H. (1996). Using language. Cambridge University Press.

Cleland, A. A., \& Pickering, M. J. (2006). Do writing and speaking employ the same syntactic representations? Journal of Memory and Language, 54, 185-198.

Coyle, J. M., \& Kaschak, M. P. (2008). Patterns of experience with verbs affect long-term cumulative structural priming. Psychonomic Bulletin \& Review, 15, 967-970.

Ferreira, V. S., Bock, K., Wilson, M. P., \& Cohen, N. J. (2008). Memory for syntax despite amnesia. Psychological Science, 19, 940-946.

Garrod, S., \& Doherty, G. (1994). Conversation, co-ordination and convention: An empirical investigation of how groups establish linguistic conventions. Cognition, 53, 181-215.

Gries, S Th. (2005). Syntactic priming: A corpus-based approach. Journal of Psycholinguistic Research, 34, 365-399.

Griffin, Z. M., \& Weinstein-Tull, J. (2003). Conceptual structure modulates structural priming in the production of complex sentences. Journal of Memory and Language, 49, 537-555.

Hartsuiker, R. J., \& Kolk, H. H. J. (1998). Syntactic persistence in Dutch. Language and Speech, 41, 143-184.

Hartsuiker, R. J., \& Westenberg, C. (2000). Word order priming in written and spoken sentence production. Cognition, 75, B27-B39.

Hartsuiker, R. J., Pickering, M. J., \& Veltkamp, E. (2004). Is syntax separate or shared between languages? Psychological Science, $15,409-414$.

Hartsuiker, R. J., Bernolet, S., Schoonbaert, S., Speybroeck, S., \& Vanderelst, D. (2008). Syntactic priming persists while the lexical boost decays: Evidence from written and spoken dialogue. Journal of Memory and Language, 58, 214-238.

Jaeger, T. F. (2008). Categorical data analysis: Away from ANOVAs (transformation or not) and towards logit mixed models. Journal of Memory and Language, 59, 434-446.

Kaschak, M. P. (2007). Long-term structural priming affects subsequent patterns of language production. Memory \& Cognition, 35, 925-937.

Kaschak, M. P., \& Borreggine, K. L. (2008). Is long-term structural priming affected by patterns of experience with individual verbs? Journal of Memory and Language, 58, 862-878.

Kaschak, M. P., Loney, R. A., \& Borreggine, K. L. (2006). Recent experience affects the strength of structural priming. Cognition, 99, B73-B82.

Kaufman, S. B., Deyoung, C. G., Gray, J. R., Jiminez, L., Brown, J., \& Mackintosh, N. (2010). Implicit learning as an ability. Cognition, 116, 321-340.
Kolers, P. A. (1976). Reading a year later. Journal of Experimental Psychology: Human Learning and Memory, 2, 554-565.

Levelt, W., \& Kelter, S. (1982). Surface form and memory in question answering. Cognitive Psychology, 14, 78-106.

Masson, M. E. J. (1984). Memory for the surface structure of sentences: Remembering with and without awareness. Journal of Verbal Learning and Verbal Behavior, 23, 579-592.

Metzing, C., \& Brennan, S. E. (2003). When conceptual pacts are broken: Partner-specific effects on the comprehension of referring expressions. Journal of Memory and Language, 49, 201213.

Mitchell, D. B. (2006). Unconscious priming after 17 years: Invulnerable implicit memory? Psychological Science, 17, 925-929.

Pickering, M. J., \& Branigan, H. P. (1998). The representation of verbs: Evidence from syntactic priming in language production. Journal of Memory and Language, 39, 633-651.

Pickering, M. J., \& Garrod, S. (2004). Toward a mechanistic psychology of dialogue. The Behavioral and Brain Sciences, 27, 169-226.

Pickering, M. J., \& Ferreira, V. S. (2008). Structural priming: A critical review. Psychological Bulletin, 134, 427-459.

Raudenbush, S. W., Bryk, A. S., Cheong, Y. F., \& Congdon, R. (2004). HLM 6: Hierarchical linear and nonlinear modeling. Scientific Software International.

Savage, C., Lieven, E., Theakston, A., \& Tomasello, M. (2003). Testing the abstractness of children's linguistic representations: Lexical and structural priming of syntactic constructions in young children. Developmental Science, 6, 557-567.

Tomasello, M. (2006). Acquiring linguistic constructions. In D. Kuhn \& R. Siegler (Eds.), Handbook of child psychology. New York: Wiley.

Tulving, E., Schacter, D. L., \& Stark, H. A. (1982). Priming effects in word fragment completion are independent of recognition memory. Journal of Experimental Psychology. Learning, Memory, and Cognition, 8, 336-341.

Tunney, R. J. (2003). Implicit and explicit knowledge decay at different rates: A dissociation between priming and recognition in artificial grammar learning. Experimental Psychology, 50, 124130.

Weiner, E. J., \& Labov, W. (1983). Constraints on the agentless passive. Journal of Linguistics, 19, 29-58.

Wheeldon, L. R., \& Smith, M. C. (2003). Phrase structure priming: A short lived effect. Language and Cognitive Processes, 18, 431442.

\section{Author's Note}

The work reported in this paper was supported in part by NSF grant 0842620. The authors would like to thank Stephen Smith, Lauren Pearson, Joel Ayoub, Evelina Tirado, Shane Lusk, Jessica Daley, and Justin Mason for their assistance in running the experiments reported here. We would also like to thank Kay Bock and two anonymous reviewers for their comments on a previous version of this manuscript. Correspondence can be addressed to Michael Kaschak, Department of Psychology, Florida State University, Tallahassee, FL 32306 (kaschak@psy.fsu.edu). 